\title{
Lateral Model Predictive Control for Over-Actuated Autonomous Vehicle
}

\author{
Gonçalo Collares Pereira ${ }^{1}$, Lars Svensson ${ }^{2}$, Pedro F. Lima ${ }^{3}$, Jonas Mårtensson ${ }^{3}$
}

\begin{abstract}
In this paper, a lateral controller is proposed for an over-actuated vehicle. The controller is formulated as a linear time-varying model predictive controller. The aim of the controller is to track a desired path smoothly, by making use of the vehicle crabbing capability (sideways movement) and minimizing the magnitude of curvature used. To do this, not only the error to the path is minimized, but also the error to the desired orientation and the control signals requests. The controller uses an extended kinematic model that takes into consideration the vehicle crabbing capability and is able to track not only kinematically feasible paths, but also plan and track over non-feasible discontinuous paths. Ackermann steering geometry is used to transform the control requests, curvature, and crabbing angle, to wheel angles. Finally, the controller performance is evaluated first by simulation and, after, by means of experimental tests on an over-actuated autonomous research vehicle.
\end{abstract}

\section{INTRODUCTION}

The increased demand for transportation lead to an increase in traffic congestions. Traffic congestion is in turn associated with decreased productivity, increased number of accidents as well as increased air pollution, and energy consumption [1], [2]. Autonomous vehicles present opportunities to mitigate these problems. In the past few years, many major vehicle manufacturers have been increasing their research in the field of autonomous vehicles and the first commercialized autonomous vehicle are already out on the roads. Autonomous driving is not a new idea and it was tested and proven possible in different competitions, such as DARPA grand and urban challenges [3], [4].

There are many different challenges associated with autonomous vehicles. A central technical challenge is lateral control of the vehicle. Where as the majority of research and development focuses on the most common 2 wheel-steering (2WS) configuration [5], [6], this paper deals with the path tracking problem for a 4 wheel-steered vehicle (4WS).

For many years, we have seen examples of 4 wheel steering vehicles in adjacent domains, such as construction vehicles and field robotics [7]. Interesting examples can also be found inthe automotive domain, such as, the DLR Robomobil [8] and the Stanford VAIL experimental vehicles [9] have been around for some time, and, recently, we have seen more examples also from industry, such as the Easymile 4-wheel steering autonomous shuttle [10].

\footnotetext{
${ }^{1}$ Integrated Transport Research Lab, Department of Automatic Control, KTH Royal Institute of Technology, SE-100 44 Stockholm, Sweden. gpcpekth.se

${ }^{2}$ Integrated Transport Research Lab, Department of Machine Design, KTH Royal Institute of Technology, SE-100 44 Stockholm, Sweden. larsvensekth.se

${ }^{3}$ Integrated Transport Research Lab and ACCESS Linnaeus Centre, Department of Automatic Control, KTH Royal Institute of Technology, SE10044 Stockholm, Sweden. pfrdal@kth. se jonasl@kth. se
}

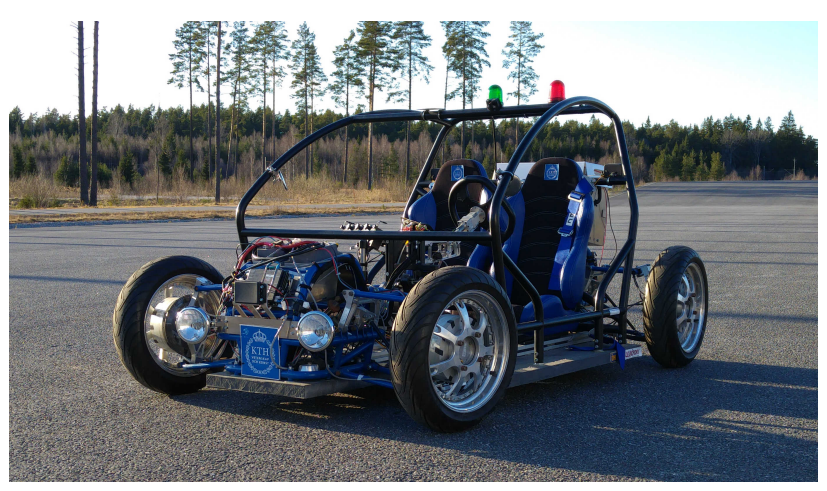

Fig. 1: Research concept vehicle (RCV).

A motivation for introducing 4WS is that it can improve yaw stability at high speed as well as manoeuvrability at low speed, as argued by Sano et al in [11]. Furthermore, 4WS allows for a type of motion called crabbing, where the vehicle moves diagonally sideways. For example, the crabbing capability of the vehicle can be used to perform lane change maneuvers with minimal yaw rate or parallel parking in tight spaces.

However, control strategies presented in literature for 4WS vehicles are usually based on thorough knowledge of vehicle dynamics and rudimentary PID control. We argue that applying model predictive control for path tracking control of a $4 \mathrm{WS}$ vehicle is a novel concept that can improve path tracking performance while maintaining a smooth and comfortable ride.

Fig. 1 presents the vehicle used for implementation and testing. The research concept vehicle (RCV) is an experimental and research platform built at KTH Royal Institute of Technology at the Integrated Transport Research Lab (ITRL) [12]. This vehicle is able to steer all four wheels independently [13].

Model predictive control (MPC) has been widely used to solve the lateral control problem of autonomous vehicles, e.g., [5], [6], [14], [15]. In [5], a MPC controller computes the front steering angle in order to follow a trajectory on slippery roads at the highest possible entry speed. There, a nonlinear MPC (NMPC) is implemented but, because of the computational burden, experimental tests could only be made at low entry speeds. To reduce the complexity of the problem, an alternative approach is presented based on successive online linearization of the vehicle model. A linear time-varying MPC (LTV-MPC) is implemented and tested, proving that the controller is able to stabilize the vehicle with a speed of up to $21 \mathrm{~m} / \mathrm{s}$ on slippery surfaces. In [6], a LTV-MPC approach is used to overcome obstacles on the road and keep 
the lane. The controller is able to avoid obstacles with an entry speed of approximately $14 \mathrm{~m} / \mathrm{s}$. There, the longitudinal and lateral control problem is separated into different optimization problems, which helps reducing the computational cost. In [14], an MPC based controller is used to stabilize a vehicle along a desired path while rejecting wind gusts and, on another scenario, perform double lane changes. It is able to stabilize the vehicle up to side wind speeds of $10.1 \mathrm{~m} / \mathrm{s}$ on the wind rejection scenario and $17 \mathrm{~m} / \mathrm{s}$ of entry speed in the double lane change maneuvers. However, due to the computational complexity of the feedback control policy, not all results are experimentally validated. More recently in [15], an LTV-MPC is used to obtain smooth and accurate path following. The optimization cost function introduces the idea of trade off between smoothness and accuracy when following a path, making use of the available free space (e.g., lane width). The controller is experimentally evaluated in a Scania mining truck and the results show that the controller is able to maintain the vehicle within $15 \mathrm{~cm}$ of the reference path both in high and low speeds.

MPC performance is highly influenced by the model used. Different models lead to different problem complexities, which ultimately lead to different computation times. In [16], kinematic and dynamic vehicle models are studied to motivate an MPC controller. The results show that the kinematic model discretized at $200 \mathrm{~ms}$ is able to perform as well as a dynamic model discretized at $100 \mathrm{~ms}$. Therefore, the authors propose MPC design based on the kinematic model instead of a higher fidelity model. In [17], different vehicle models for lateral vehicle dynamics are described, which are then used by the author to develop and validate controllers to maintain a vehicle in the center of its road lane boundaries.

The work presented in this paper extends the previous research. A kinematic vehicle model is extended to take into consideration the extra degree of freedom of the RCV and used to design an LTV-MPC for lateral control of the vehicle. The controller makes use of crabbing angle and curvature requests to follow a desired path in a smooth and comfortable manner. Furthermore, the controller is subject to a two-phase validation. First, the controller is validated in a simulation environment that uses an accurate vehicle model of the RCV. Then, the controller is implemented in the RCV and its performance evaluated through field tests at a local test site at KTH, Stockholm, Sweden.

This paper is organized as follows: in Section II, the vehicle's steering concept is presented; in Section III, the vehicle models used are presented; in Section IV, the problem of path following is addressed by developing an LTV-MPC controller; in Section V, both simulation and experimental results are presented and compared; finally, in Section VI, concluding remarks are presented and future work outlined.

\section{SteEring CONCEPT}

Ackerman steering is a geometric arrangement for minimizing tire slip when a vehicle is turning. This is achieved by choosing steering angles such that all wheels are tangent to a set of concentric circles. Assuming planar motion and small tire slip, the vehicle rotates about the center of the

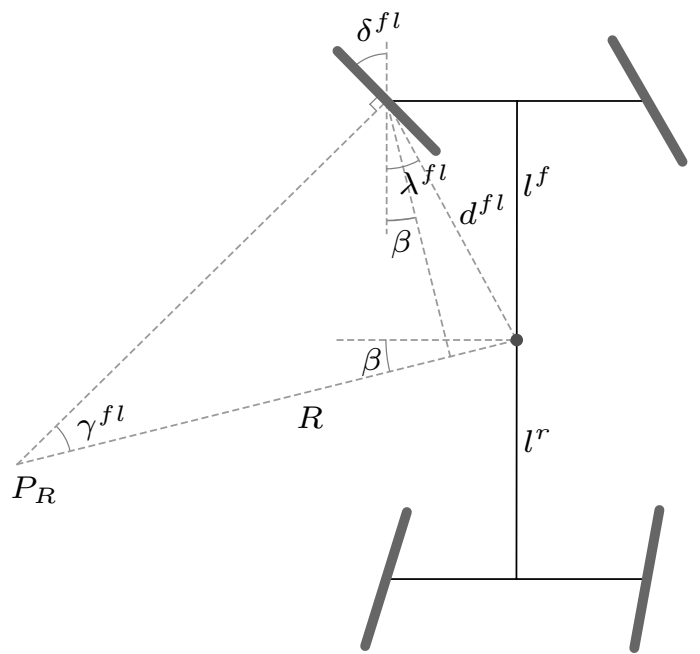

Fig. 2: Geometric relations between $\delta^{f l}$ and point of rotation, $P_{R}$.

circle, called the instantaneous center of rotation $P_{R}$, i.e., the vehicle turns with the curvature $\kappa=1 / R$, where $R$ is the distance between the rotational center of the vehicle and $P_{R}$.

For $2 \mathrm{WS}$ vehicles, $P_{R}$ must be placed on a line that extends the rear axle, but for 4WS vehicles, it can be placed at an arbitrary point in the plane, provided that steering angle constraints for each individual wheel are respected. Placing $P_{R}$ in front or behind the point of rotation causes the vehicle to crab, thus introducing an additional degree of freedom.

$P_{R}$ is expressed in polar coordinates in the vehicle frame, see Fig. 2. The argument $\beta$ corresponds to the angle between the vehicle's velocity vector and the forward direction of the vehicle chassis, i.e., the crab angle.

Based on a requested curvature $\kappa$ and a requested crabbing angle $\beta, P_{R}$ is fully determined, and from basic trigonometry, the individual steering angles are obtained. For example, the front left wheel angle $\delta^{f l}=\beta+\gamma^{f l}$, where

$$
\gamma^{f l}=\operatorname{atan}\left(\frac{d^{f l} \cos \left(\lambda^{f l}-\beta\right)}{R-d^{f l} \sin \left(\lambda^{f l}-\beta\right)}\right) .
$$

Using this formulation, the vehicle is able to crab and curve simultaneously, while maintaining the Ackerman steering geometry.

\section{Modelling}

In this section, two different vehicle models are presented. Namely, an extended kinematic model used for vehicle prediction within the MPC framework, and a more complex and accurate dynamic vehicle model for controller validation in simulation.

\section{A. Controller Vehicle Model}

The continuous-time differential equations of the vehicle model can be obtained by adding the crabbing angle to a standard kinematic model. The vehicle model discretized 
version is

$$
\begin{aligned}
& X_{i+1}=X_{i}+T_{s} v_{i} \cos \left(\Psi_{i}+\beta_{i}\right), \\
& Y_{i+1}=Y_{i}+T_{s} v_{i} \sin \left(\Psi_{i}+\beta_{i}\right), \\
& \Psi_{i+1}=\Psi_{i}+T_{s} v_{i} \kappa_{i},
\end{aligned}
$$

where, $X, Y$, and $\Psi$ are the vehicle's coordinates and orientation, respectively, in the world frame, $v$ is the vehicle velocity, $T_{s}$ is a sampling time, and $\beta$ and $\kappa$ are the control signals, crabbing angle and curvature, respectively. The index $i$ denotes the time instant at which the variable is being considered.

From (1), it is possible to rotate and translate the states to local vehicle coordinates, i.e., the origin of the Cartesian frame corresponds to the center of gravity pose of the vehicle, and write the vehicle model states as $z=[x, y, \psi]^{T}$, and the control signals as $u=[\kappa, \beta]^{T}$. As mentioned before, this model is used to predict the vehicle motion in the MPC framework for path following. Since the vehicle model is clearly nonlinear, it is linearized considering a reference path composed of $N$ points, $z_{j}^{\text {ref }}=\left[x_{j}^{\text {ref }} ; y_{j}^{\text {ref }} ; \psi_{j}^{\text {ref }}\right]$ and $u_{j}^{\text {ref }}=\left[\kappa_{j}^{\text {ref }} ; \beta_{j}^{\text {ref }}\right]$, where, $j=1, \ldots, N$. The reference points are discretized according to the velocity profile and the sampling time. Finally, the linearized vehicle model states, can be expressed as

$$
\tilde{z}_{j}=A_{j} \tilde{z}_{j}+B_{j} \tilde{u}_{j}
$$

where, $\tilde{z}_{j}=z_{j}-z_{j}^{\text {ref }}, \tilde{u}_{j}=u_{j}-u_{j}^{\text {ref }}$, and $A_{j}$ and $B_{j}$ can be expressed as

$$
\begin{aligned}
A_{j} & =\left[\begin{array}{ccc}
1 & 0 & -T_{s} v_{j}^{\text {ref }} \sin \left(\psi_{j}^{\text {ref }}+\beta_{j}^{\text {ref }}\right) \\
0 & 1 & T_{s} v_{j}^{\text {ref }} \cos \left(\psi_{j}^{\text {ref }}+\beta_{j}^{\text {ref }}\right) \\
0 & 0 & 1
\end{array}\right], \\
B_{j} & =\left[\begin{array}{ccc}
0 & -T_{s} v_{j}^{\text {ref }} \sin \left(\psi_{j}^{\text {ref }}+\beta_{j}^{\text {ref }}\right) \\
0 & T_{s} v_{j}^{\text {ref }} \cos \left(\psi_{j}^{\text {ref }}+\beta_{j}^{\text {ref }}\right) \\
T_{s} v_{j}^{\text {ref }} & 0
\end{array}\right] .
\end{aligned}
$$

\section{B. Simulation Vehicle Model}

The vehicle model used in simulation is based upon the models used in [5], [14], [16], [17] and can be obtained from Fig. 3,

$$
\begin{aligned}
& X_{i+1}=X_{i}+T_{s}\left(v_{x_{i}} \cos \left(\Psi_{i}\right)-v_{y_{i}} \sin \left(\Psi_{i}\right)\right), \\
& Y_{i+1}=Y_{i}+T_{s}\left(v_{x_{i}} \sin \left(\Psi_{i}\right)+v_{y_{i}} \cos \left(\Psi_{i}\right)\right), \\
& \Psi_{i+1}=\Psi_{i}+T_{s} \omega_{i}, \\
& v_{x_{i+1}}=v_{x_{i}}+T_{s}\left(\frac{F_{x_{i}}-F_{r}-F_{a_{i}}}{M}+v_{y_{i}} \omega_{i}\right), \\
& v_{y_{i+1}}=v_{y_{i}}+T_{s}\left(\frac{F_{y_{i}}}{M}-v_{x_{i}} \omega_{i}\right) \\
& \omega_{i+1}=\omega_{i}+T_{s} \frac{l^{f}\left(F_{y_{i}}^{f r}+F_{y_{i}}^{f l}\right)-l^{r}\left(F_{y_{i}}^{r r}+F_{y_{i}}^{r l}\right)}{I} .
\end{aligned}
$$

where, $v_{x}$ and $v_{y}$ are the vehicle's longitudinal and lateral velocities, in the vehicle's local frame, $\omega$ is the yaw rate of the vehicle, $M=m+m_{I}$ is the total mass, where, $m$ is the vehicle mass and $m_{I}$ is the equivalent mass of rotating parts due to inertia, $I$ is the vehicle inertia around the z-axis, and $l^{f}$ and $l^{r}$ are the distances from the front and rear wheels to the center of gravity $(\mathrm{CoG})$, respectively. The index $i$ denotes the discrete time instant at which the variable is being considered. Without considering the time instant, $F_{x}=F_{x}^{f r}+F_{x}^{f l}+F_{x}^{r r}+F_{x}^{r l}$ and

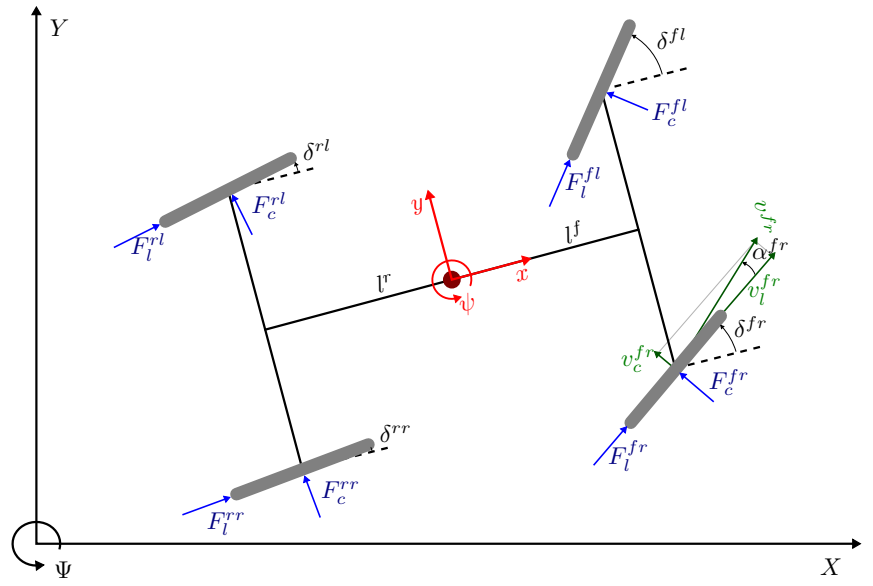

Fig. 3: Illustration of the vehicle model used in simulation. The wheels are presented in gray, the forces on each wheel in blue, the velocities for the front right wheel are presented in green, and the center of gravity and the axis for the local frame of the vehicle are presented in red.

$F_{y}=F_{y}^{f r}+F_{y}^{f l}+F_{y}^{r r}+F_{y}^{r l}$ are the longitudinal and lateral forces acting on $\mathrm{CoG}$ of the vehicle, $F_{a}=\frac{1}{2} \rho C_{d} A_{f} v_{x}^{2}$ is the air drag force, where, $\rho$ is the mass density of air, $C_{d}$ is the aerodynamic drag coefficient, $A_{f}$ is the frontal area of the vehicle and, finally, $F_{r}=f_{\text {roll }} m g \cos (\theta)$ is the rolling resistance force, where, $f_{\text {roll }}$ is the roll resistance coefficient, $g$ is the gravitational acceleration and $\theta$ is the road inclination angle. The notation $(.)^{f r, f l, r r, r l}$ corresponds, respectively, to front right, front left, rear right, and rear left wheel.

For all wheels a constant normal tire load is assumed, i.e., $F_{z}=$ constant. The forces acting on the CoG due to the front right wheel are

$$
\begin{aligned}
& F_{x}^{f r}=F_{l}^{f r} \cos \left(\delta^{f r}\right)-F_{c}^{f r} \sin \left(\delta^{f r}\right), \\
& F_{y}^{f r}=F_{l}^{f r} \sin \left(\delta^{f r}\right)-F_{c}^{f r} \cos \left(\delta^{f r}\right),
\end{aligned}
$$

where, $\delta$ is the angle of the wheel, $F_{l}=\frac{T}{r}$ is the longitudinal force, where $T$ is the torque and $r$ is the radius, and $F_{c}=C \alpha$ is the lateral force of the tire, where $C$ is the cornering stiffness and $\alpha$ is the slip angle that can be expressed for the front and rear wheels as

$$
\begin{aligned}
& \alpha^{f r, f l}=\delta^{f r, f l}-\arctan \left(\frac{v_{y}+l^{f r, f l} \omega}{v_{x}}\right), \\
& \alpha^{r r, r l}=\delta^{r r, r l}-\arctan \left(\frac{v_{y}-l^{r r, r l} \omega}{v_{x}}\right) .
\end{aligned}
$$

\section{Model Predictive Controller}

This section addresses the problem of path following for a 4WS vehicle. An LTV-MPC is formulated to perform path tracking while maintaining a smooth and comfortable ride by making use of the additional degree of freedom, the crabbing angle.

The main objective of the controller is to follow a given path, even if the path is not planned with a $4 \mathrm{WS}$ vehicle dynamics in mind. Therefore, the problem of reference generation needs to be addressed. 


\section{A. Reference Generation}

The generation of the reference path points and control signals is a crucial step, because the linearized vehicle model is only valid in the vicinity of the linearization points and is highly dependent on their choice. Furthermore, a good choice of the reference control signals and path points contributes to a faster convergence of the solver and a smaller computation time.

Before explaining the reference generation, it is important to define and introduce some terms. Vehicle orientation is defined as the yaw $(\Psi)$ of the vehicle. Road direction is defined by the tangent of the road at any given point. For example, the orientation of a regular vehicle when performing a lane change on a straight road does not match the path direction, it matches the tangent of the traveled path.

Assuming the path was generated for a regular vehicle, the expected orientation $(\Psi)$ at each point corresponds to the direction of movement of the vehicle. So, this type of path does not explicitly use the extra degree of freedom $\beta$ and, if no further information is provided, this term is only used to correct error and deal with unfeasible paths. However, if the road/desired direction is given to the controller, the desired orientation at each point becomes the direction of the road, which ultimately leads to a smoother and more comfortable ride, because the vehicle follows the path using a combination of curvature and crabbing, and not, for example, using only curvature to perform a lane change.

Independently of receiving the road direction or not, the reference control signals are constrained to limit values. The constrained path $\left(z_{\text {calc }}^{\text {ref }}\right)$ is created by trying to follow as much as possible the direction of the original path $\left(z_{\text {true }}^{\text {ref }}\right)$, which in the case presented in Fig. 4 leads to maximum curvature at the beginning of the step, because during the step the path direction is $\pi / 2$ radians, and, then, minimum curvature to get the direction back to 0 radians to match the path.

To resume, the original path $\left(z^{\text {ref }}\right)$ is in the world frame and is used to compute the current error to the path $(\tilde{z})$, note that the controller controls over a local frame not the world frame. So, $z^{\text {ref }}$ is transformed for the controller into $z_{\text {true }}^{\text {ref }}$, which is the original path in local frame coordinates and discretized properly taking in consideration the control horizon. From $z_{\text {true }}^{\text {ref }}$ and the reference control signals required to follow that path, $z_{\text {calc }}^{\text {ref }}$ is created by constraining the control signals.

Limiting the control signals, makes the reference path points feasible, allowing for a faster convergence of the solver, since the reference points always respect the maximum and minimum control signals constraints. On the other hand, saturating the control signals, has the disadvantage of changing the reference path points, which ultimately would make the vehicle follow a path that is different from the original path. To deal with this, the cost function is modified to account for future predicted error, which is presented in Fig. 4. In other words, a term is added to the cost function to account for the difference between the desired path sent to the controller and the reference path used to linearize the vehicle model. This is further explained in the next subsection.

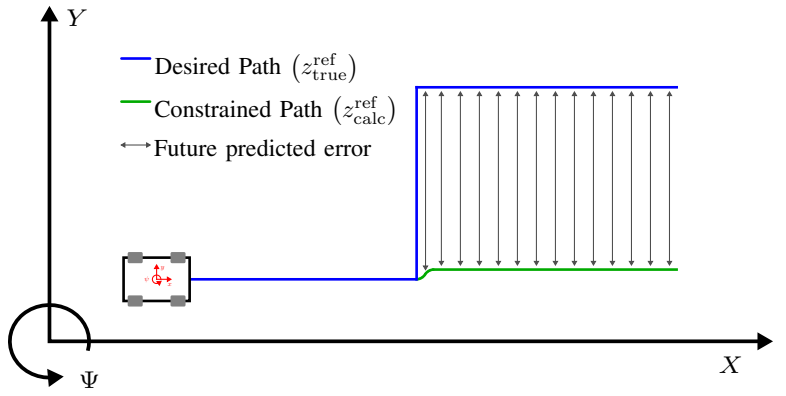

Fig. 4: Illustration of the constrained path created because of the saturation of the control signal while generating the references. The future predicted error in each point is the difference between both paths.

\section{B. Problem Formulation}

The objective of the controller is not only to track a given path, but also to track the direction of the road, by using curvature and crabbing angle requests. The initial controller can be formulated as

$$
\begin{array}{cl}
\min _{\tilde{u}} & \|\tilde{z}\|_{Q^{z}}^{2}+\left\|\psi-\psi^{\mathrm{road}}\right\|_{Q^{\mathrm{road}}}^{2}+\|u\|_{Q^{u}}^{2} \\
\text { s.t. } & \tilde{z}_{i+1}=A \tilde{z}_{i}+B \tilde{u}_{i}, i=1, \ldots, H_{p} \\
& \tilde{z}_{i}=z_{i}-z_{i}^{\mathrm{ref}}, i=1, \ldots, H_{p}, \\
& \tilde{u}_{i}=u_{i}-u_{i}^{\mathrm{ref}}, i=1, \ldots, H_{c} \\
& u_{\min } \leq u \leq u_{\max }, \\
& \Delta u_{\min } \leq \Delta u \leq \Delta u_{\max }
\end{array}
$$

where, $\|\tilde{z}\|_{Q^{z}}^{2}=\tilde{z}^{T} Q_{z} \tilde{z}$ and $Q^{z}=\operatorname{diag}\left(Q^{x}, Q^{y}, Q^{\psi}\right)$ are the weights for the lateral states deviations, $\psi^{\text {road }}$ is the road direction and if not given to the controller it is $\psi^{\text {road }}=\psi^{\text {ref }}, Q^{\text {road }}$ is the weight for the road direction deviation, $Q^{u}=\operatorname{diag}\left(Q^{\kappa}, Q^{\beta}\right)$ are the weights for the control signals, $z=[x, y, \psi]^{T} \in \mathbb{R}^{3 \times\left(H_{c}+H_{p}\right)}$ are the vehicle model states used and $u=[\kappa, \beta]^{T} \in \mathbb{R}^{2 \times H_{c}}$ are the control signals. The notation $\Delta a$ represents the rate of $a$ and is approximated by $\frac{a_{i}-a_{i-1}}{T_{s}}$. Finally, $H_{c}$ is the control horizon and $H_{p}$ is the prediction horizon.

The control horizon is defined to be shorter than the prediction horizon, which significantly reduces the complexity of the problem, while maintaining a long state prediction horizon. In order to do this, the last control signal is maintained constant throughout the states that do not have a time-matching control signal [18], i.e., the control signals $u_{H_{c}+1}, \ldots, u_{H_{p}}=u_{H_{c}}$.

As mentioned before, because of the reference generation, it is necessary to modify the cost function to account for the reference change from non feasible to feasible control signal references. To do this, the future predicted error term, depicted in Fig. 4, $\tilde{z}^{\text {fut }}=z_{\text {calc }}^{\text {ref }}-z_{\text {true }}^{\text {ref }}$, is added and the term $\|\tilde{z}\|_{Q^{z}}^{2}$ is modified to $\left\|\tilde{z}+\tilde{z}^{\text {fut }}\right\|_{Q^{z}}^{2}$. By also adding the future predicted error to the road direction term, the final 
TABLE II: Weights

TABLE I: Constraints

\begin{tabular}{l|l}
\hline$|\beta|$ & $0.1222 \mathrm{rad}$ \\
$|\kappa|$ & $0.1579 \mathrm{~m}^{-1}$ \\
$|\Delta \beta|$ & $0.2318 \mathrm{rad} / \mathrm{s}$ \\
$|\Delta \kappa|$ & $0.1500 \mathrm{~m}^{-1} \mathrm{~s}^{-1}$
\end{tabular}

\begin{tabular}{l|l}
\hline$Q^{x}$ & 10 \\
$Q_{f}^{x}$ & 100 \\
$Q^{y}$ & 50 \\
$Q_{f}^{y}$ & 500 \\
$Q^{\psi}$ & 0 \\
$Q_{f}^{\psi}$ & 0 \\
$Q^{\text {road }}$ & 100 \\
$Q_{f}^{\text {road }}$ & 1000 \\
$Q^{\kappa}$ & 1000 \\
$Q^{\beta}$ & 100 \\
\hline
\end{tabular}

formulation can be written as

$$
\begin{array}{ll}
\min _{\tilde{u}} & \left\|\tilde{z}+\tilde{z}^{\text {fut }}\right\|_{Q^{z}}^{2}+\left\|\tilde{\psi}^{\text {road }}+\tilde{\psi}^{\text {fut }}\right\|_{Q^{\text {road }}}^{2} \\
& +\left\|\tilde{u}+u^{\text {ref }}\right\|_{Q^{u}}^{2} \\
\text { s.t. } & \tilde{z}_{i+1}=A \tilde{z}_{i}+B \tilde{u}_{i}, i=1, \ldots, H_{p}, \\
& \tilde{z}_{i}=z_{i}-z_{i}^{\text {ref }}, i=1, \ldots, H_{p}, \\
& \tilde{u}_{i}=u_{i}-u_{i}^{\text {ref }}, i=1, \ldots, H_{c}, \\
& \tilde{\psi}_{i}^{\text {road }}=\psi_{i}-\psi_{i}^{\text {road }}, \\
& u_{\text {min }}-u^{\text {ref }} \leq \tilde{u} \leq u_{\max }-u^{\text {ref }}, \\
& \Delta u_{\min }-\Delta u^{\text {ref }} \leq \Delta \tilde{u} \leq \Delta u_{\max }-\Delta u^{\text {ref }},
\end{array}
$$

where, the optimal control signal is $u^{\star}=\tilde{u}+u^{\text {ref }}$. This problem is trivial to cast as a quadratic programming problem (QP), which is the formulation used to solve the optimization problem. Further information on how to cast the problem as QP can be found, for example, in [19]. Only the first elements of the optimal control signal are used as a control input, on the next iteration step the problem (11) is solved again and new control signals are computed.

\section{Controller Specifications}

This section presents the controller tuning procedure.

1) Frequency: The controller rate is set up based on the low level controllers of the vehicle, these controllers run with a period of $T_{\text {LowLevel }}=0.03 \mathrm{~s}$ and send the control command to the controllers of each actuator, so to allow the actuators to reach the desired point and to allow them to actuate with that signal, the MPC rate is set with a period of $T_{\mathrm{MPC}}=0.09 \mathrm{~s}$.

2) Horizon: The horizons are set to be as large as possible, taking into consideration the time it takes to solve the problem with the hardware used. The control horizon is set to $H_{c}=15$ and the prediction horizon to $H_{p}=45$, which corresponds to $1.35 \mathrm{~s}$ and $4.05 \mathrm{~s}$, respectively. This leads to an average turnaround time of $0.0653 \mathrm{~s}$ during the experiment. This time takes into consideration not only the minimization problem, but also all the other tasks the computer has to perform during one lateral MPC computation, i.e., the longitudinal MPC controller, low-level controllers, sensor reading, communication, etc.

3) Constraints: The constraints imposed on the problem and presented on Table I are mainly mechanical, but by limiting the steering actuators rate, a comfort limit is also

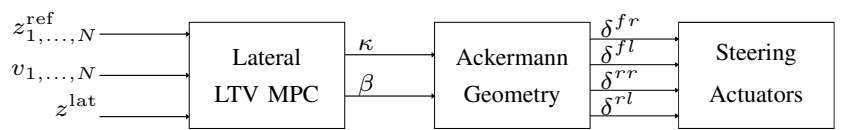

Fig. 5: Block diagram of the control algorithm implemented in the vehicle.

imposed. Furthermore, aggressive steering, results in a higher "wear and tear", which is undesirable because it shortens the actuators lifetime.

4) Weights: The weights presented in Table II are tuned to obtain the desired performance. The objective of the controller is to track a path so it is mostly important to minimize the $y^{\text {ref }}$ deviation. Furthermore, as previously mentioned, it is preferable to track the road direction rather than the path direction, so the gains are set accordingly. Finally, the relation between the crabbing angle and curvature weights is set so that crabbing is preferable. This is more comfortable since the occupants experience less rotational movement.

\section{Control Architecture}

Fig. 5 presents the block diagram of the control algorithm implemented in the RCV. The information fed to the controller is the future velocity profile, the desired path, and the current state of the vehicle. The delay between request and actuation is neglected. The curvature and crabbing angle request are converted to steering angles for each wheel according to the dimensions of the vehicle and the Ackermann steering geometry.

The LTV-MPC is implemented in MATLAB/Simulink and the optimization problem is solved using CVXGEN, which is a fast custom optimization solver [20]. Using [21] custom Ccode is created for the specific LTV MPC problem. Hardware wise, a real-time system for performing fast function prototyping, MicroAutoBoxII 1401/1513 from dSPACE, is used. Furthermore, the current state of the vehicle is obtained from a Kalman filter implemented on a VBOX 3I SL Racelogic, which has an IMU and a dual antenna GPS without RTK corrections.

\section{RESULTS}

In this section, both simulation and experimental results, obtained with the RCV are described. First, the test scenario used is presented. After, the controller is validated in simulation using the more complex force model presented in Chapter III. Finally, the results obtained with the RCV are presented and compared against the simulation.

\section{A. Scenario}

The scenario used aims to showcase the capabilities and advantages of the controller. The scenario consists of a lane change on a straight road. The lanes considered have a width of 3.5 meters and the desired path on each lane is always the centerline of the lane.

The lane change is performed using two different paths. According to [15], a path with linear-varying curvature composed of clothoids is smooth and comfortable, so the first path is composed of this type of curves with a curvature rate of $0.0366 \mathrm{~m}^{-1} \mathrm{~s}^{-1}$, which, sampled every $0.09 \mathrm{~s}$ at a speed of $2.5 \mathrm{~m} / \mathrm{s}$, leads to a lane change in approximately 


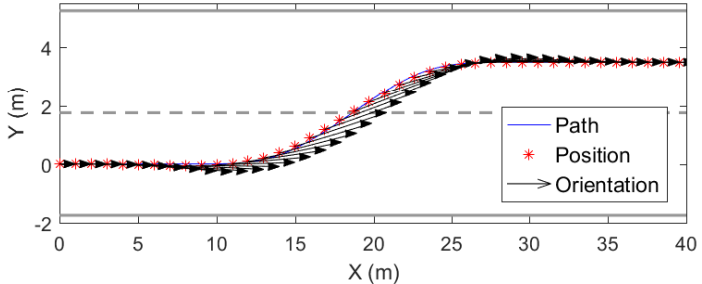

(a) Position and orientation of the vehicle. The orientation of the vehicle does not match the direction of the path.
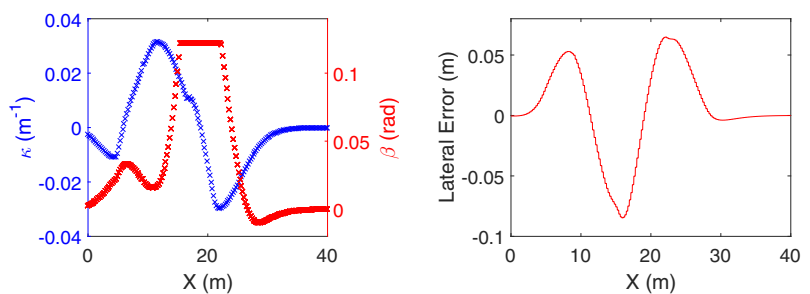

(b) Curvature and crabbing angle (c) Lateral error of the vehicle in requests. The crabbing angle sat- relation to the path. urates during the lane change.

Fig. 6: Simulation result for the clothoidal path.

$18 \mathrm{~m}$. The second path is a non feasible path, which does not account for the vehicle's movement in between lanes. The path is discontinuous and instantaneously changes to the other lane at a certain point, which allows to showcase one of the big advantages of using MPC, since it is not only able to follow a continuous path but also to predict and perform non feasible paths in a smooth and accurate manner, assuming that it is properly tuned.

\section{B. Simulation}

In Fig. 6, the lane change with the clothoidal path is presented. As it is visible in the Fig. 6a, the vehicle performs the path by using mostly the crabbing motion, but, since this motion is constrained some curvature is requested also. This is visible in Fig. 6b, where it is verified that the maximum and minimum curvature used are, respectively, $0.0316 \mathrm{~m}^{-1}$ and $-0.0296 \mathrm{~m}^{-1}$, compared to the maximum and minimum of the path, respectively, $0.0794 \mathrm{~m}^{-1}$ and $-0.0724 \mathrm{~m}^{-1}$. Furthermore, the average magnitude of the curvature rate during the actual lane change is $0.0119 \mathrm{~m}^{-1} \mathrm{~s}^{-1}$, which is smaller than the constant rate used to create the path. So, the obtained motion is more comfortable than the original planned path, because the occupants experience less rotational movement. Fig. $6 \mathrm{c}$ presents the lateral error obtained during the simulation. The error is associated with the use of the crabbing motion and happens because the path is not planned for a $4 \mathrm{WS}$ vehicle, and it is not possible to describe exactly the same path when using crabbing or curvature, since, the first leads to a straight path and the second leads to a curved path. A constant velocity of $2.5 \mathrm{~m} / \mathrm{s}$ is used. This velocity is chosen because it is not possible to test the real vehicle at higher speeds due to safety limitations at the test site.

In Fig. 7, the lane change with the discontinuous path is presented. As it is visible in the Fig. 7a, the controller is able to predict and perform the path by using mostly the

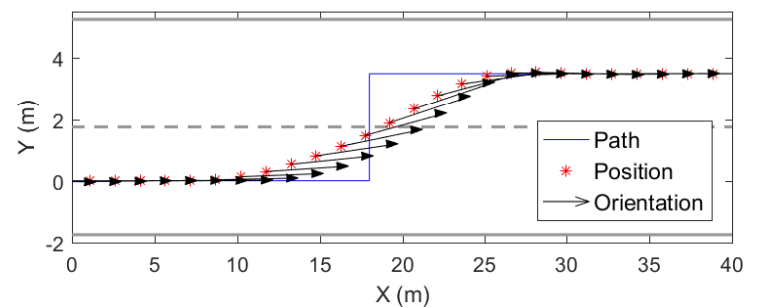

(a) Position and orientation of the vehicle. The discontinuity in the path is smoothen out and starts being corrected earlier than when it happens.
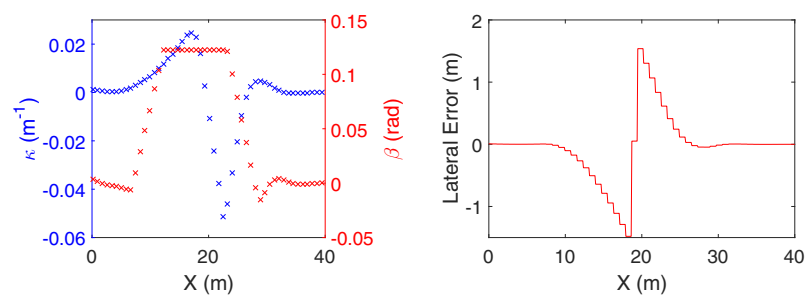

(b) Curvature and crabbing angle (c) requests.

(c) Lateral error of the vehicle in

Fig. 7: Simulation result for the discontinuous path.

crabbing motion. This is also visible in Fig. $7 b$, where it is verified that the maximum and minimum curvature used are, respectively, $0.0246 \mathrm{~m}^{-1}$ and $-0.0513 \mathrm{~m}^{-1}$, and the maximum and minimum curvature rate used by the vehicle are, respectively, $0.1445 \mathrm{~m}^{-1} \mathrm{~s}^{-1}$ and $-0.15 \mathrm{~m}^{-1} \mathrm{~s}^{-1}$. Fig. 7c presents the lateral error obtained during the simulation. The error is more or less symmetrical, which is the expected behavior considering the path. A constant velocity of $9 \mathrm{~m} / \mathrm{s}$ is maintained by a longitudinal controller, this velocity is higher than the one used on the previous simulation to demonstrate that the controller is able to perform well at higher speeds. The controller gains used in this simulation are tuned to deal with the discontinuity and, since, the controller is supposed to always receive a kinematically feasible path, this result is not tested with the real vehicle. The controller does not explicitly handle obstacle avoidance, because it is assumed that the path planner takes care of creating a feasible non colliding path. For this scenario, the assumption is extended and it is assumed that the path in between lanes is free, future work will be considered to address this issue.

\section{Experimental Results}

In Fig. 8, the lane change with the clothoidal path performed at $2.5 \mathrm{~m} / \mathrm{s}$ is presented. The results were obtained using the RCV, and performing the scenario in between buildings, with snowy/icy conditions, and using motorcycle tires without chains or studs. As it is visible in the Fig. 8a, the vehicle performs the path by using mostly the crabbing motion. The maximum and minimum curvature used are, respectively, $0.0447 \mathrm{~m}^{-1}$ and $-0.0564 \mathrm{~m}^{-1}$, which are smaller than the maximum and minimum curvature of the path. Furthermore, the average magnitude of the curvature rate during the actual lane change is $0.0236 \mathrm{~m}^{-1} \mathrm{~s}^{-1}$, which is smaller than the constant rate used to perform the lane 


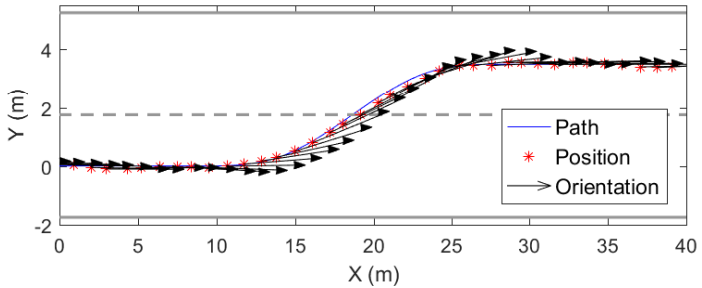

(a) Position and orientation of the vehicle. The oscillations before and after the lane change are mostly from GPS drift and were not noticeable during the experiment.
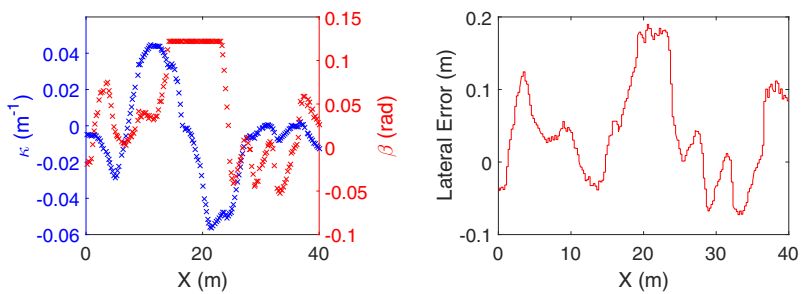

(b) Curvature and crabbing angle (c) Lateral error of the vehicle requests. The GPS drift leads to in relation to the path. Note, for some $\beta$ and $\kappa$ but the vehicle example, the jump in lateral error converged always to the path. that occurred at $36.57 \mathrm{~m}$, which happens because of the GPS.

Fig. 8: Experimental result for the clothoidal path.

change.

The error presented in Fig. 8b, is not only associated with the use of the crabbing motion as in the simulation, but also with GPS drift and the complex dynamics of the vehicle. The actuators installed on the RCV are worn and not very precise, which lead to bigger errors. Furthermore, a rough calibration of the wheel alignment is done a priori, which lead to unmodeled behavior. The GPS drift is bigger at the beginning and at the end of the path, when the vehicle was closer to buildings. This drift is clear around $36.57 \mathrm{~m}$, where with the speed, orientation, and crabbing angle the vehicle $\mathrm{had}$, it is impossible for it to move $0.6 \mathrm{~m}$ sideways as the results show.

\section{CONCLUSiOnS AND FUture WORK}

In this paper, an LTV-MPC for a 4WS vehicle is presented. The controller makes use of the additional degree of freedom of the vehicle to track a path in a smoother and comfortable manner. To do this, not only the error to the path is minimized, but also the error to the road/desired direction. This way, the vehicle can crab, i.e., move sideways, to track the path, even when the desired orientation is not tangent to the path.

The controller is able to predict and perform the path using not only curvature requests but also crabbing angle requests, even for paths planned only assuming curvature. The vehicle was able to track a clothoidal lane change on icy/snowy conditions. It performed the lane change in a smooth and accurate manner, saturating the use of $\beta$, using a maximum curvature magnitude of $0.0564 \mathrm{~m}^{-1}$ and an average curvature rate magnitude of $0.0236 \mathrm{~m}^{-1} \mathrm{~s}^{-1}$, both smaller than the intended in the planned path.
The long term research goal of the RCV is to demonstrate fully autonomous and connected mobility in city environments. With respect to future controller development, this brings requirements for integrating with the larger scale system. For example, this may involve introducing state constraints provided by other functions in the system, in order to guarantee collision free operation. This also implies doing further tests at different speeds, to guarantee robustness and the generality of the solution proposed.

\section{REFERENCES}

[1] B. van Arem, C. J. G. van Driel, and R. Visser, "The impact of cooperative adaptive cruise control on traffic-flow characteristics," IEEE Transactions on Intelligent Transportation Systems, vol. 7, no. 4, pp. 429-436, December 2006.

[2] J. Ploeg, S. Shladover, H. Nijmeijer, and N. van de Wouw, "Introduction to the special issue on the 2011 grand cooperative driving challenge," IEEE Transactions on Intelligent Transportation Systems, vol. 13, no. 3, pp. 989-993, September 2012.

[3] S. Thrun et al., "Stanley: The robot that won the darpa grand challenge," Journal of Field Robotics, vol. 23, no. 9, pp. 661-692, June 2006.

[4] C. Urmson et al., "Autonomous driving in urban environments: Boss and the urban challenge," Journal of Field Robotics, vol. 25, no. 8, pp. 425-466, 2008. [Online]. Available: http://dx.doi.org/10.1002/rob.20255

[5] P. Falcone, F. Borrelli, J. Asgari, H. E. Tseng, and D. Hrovat, "Predictive active steering control for autonomous vehicle systems," IEEE Transactions on Control Systems Technology, vol. 15, no. 3, pp. 566-580, 52007.

[6] V. Turri, A. Carvalho, H. E. Tseng, K. H. Johansson, and F. Borrelli, "Linear model predictive control for lane keeping and obstacle avoidance on low curvature roads," in Intelligent Transportation Systems (ITSC), 2013 16th International IEEE Conference on, 10 2013, pp. 378-383.

[7] T. Bak and H. Jakobsen, "Agricultural robotic platform with four wheel steering for weed detection," Biosystems Engineering, vol. 87, no. 2, pp. 125 - 136, 2004. [Online]. Available: http://www.sciencedirect.com/science/article/pii/S153751100300196X

[8] T. Bünte, L. M. Ho, C. Satzger, and J. Brembeck, "Central vehicle dynamics control of the robotic research platform robomobil,' ATZelektronik worldwide, 2014.

[9] Stanford volkswagen automotive innovation lab. [Online]. Available: http://web.stanford.edu/group/vail/

[10] Easymile mobility solution. [Online]. Available: http://easymile.com/

[11] S. Sano, Y. Furukawa, and S. Shiraishi, "Four wheel steering system with rear wheel steer angle controlled as a function of steering wheel angle," SAE Technical Paper, Tech. Rep., 1986.

[12] Research concept vehicle. [Online]. Available: https://www.itrl.kth.se/research/itrl-labs/rcv-1.476469

[13] O. Wallmark et al., "Design and implementation of an experimental research and concept demonstration vehicle," in 2014 IEEE Vehicle Power and Propulsion Conference (VPPC), Oct 2014, pp. 1-6.

[14] T. Keviczky, P. Falcone, F. Borrelli, J. Asgari, and D. Hrovat, "Predictive control approach to autonomous vehicle steering," in American Control Conference, 2006, June 2006, pp. 6 pp.-.

[15] P. F. Lima, M. Trincavelli, M. Nilsson, J. Mrtensson, and B. Wahlberg, "Experimental evaluation of economic model predictive control for an autonomous truck," in 2016 IEEE Intelligent Vehicles Symposium (IV), June 2016, pp. 710-715.

[16] J. Kong, M. Pfeiffer, G. Schildbach, and F. Borrelli, "Kinematic and dynamic vehicle models for autonomous driving control design," in 2015 IEEE Intelligent Vehicles Symposium (IV), June 2015, pp. 1094 1099 .

[17] R. Rajamani, Vehicle Dynamics and Control.

[18] J. Rossiter, Model-Based Predictive Control: A Practical Approach, ser. Control Series. CRC Press, 2003. [Online]. Available: https://books.google.se/books?id=owznQTI-NqUC

[19] F. Kühne, J. Gomes, and W. Fetter, "Mobile robot trajectory tracking using model predictive control," in II IEEE latin-american robotics symposium, 2005.

[20] J. Mattingley and S. Boyd, "Cvxgen: A code generator for embedded convex optimization," Optimization and Engineering, vol. 13, no. 1, pp. 1-27, 2012.

[21] Cvxgen. [Online]. Available: http://cvxgen.com/docs/index.html 